\title{
Meditação on-line: uma estratégia educativa para promoção da saúde mental no contexto da pandemia por COVID-19
}

Online meditation: na educational strategy to promote mental health in the contexto of the COVID19 pandemic

Meditación em línea: uma estratégia educativa para promover la salud mental en el contexto de la pandemia COVID-19

\section{Resumo}

Este estudo objetiva relatar a experiência do ensino da meditação on-line como prática promotora da saúde mental durante o isolamento social da pandemia por COVID-19. Trata-se de relato de experiência sobre uma das atividades desenvolvidas pelo Projeto de Extensão para Estratégias de Promoção da Saúde Mental na Universidade Federal Fluminense. Devido à pandemia por COVID- 19, as ações tem sido realizadas de maneira remota, neste caso, a meditação. Foram realizados dois ciclos de meditação da Abundância e dois ciclos de Ho'oponopono, com 21 dias em cada ciclo. As atividades da meditação da abundância contaram com a participação de 225 pessoas, e as atividades de Ho'oponopono contaram com a participação de 357 pessoas. O feedback dos participantes sobre a experiência trouxe a percepção da melhora da ansiedade e do medo da contaminação por COVID-19, além de aprendizado profundo com foco no autoconhecimento. Foi possível concluir que a meditação é um recurso relevante para promoção da saúde mental, principalmente em períodos de distanciamento social.

Palavras-chave: Meditação; Qualidade de vida; Saúde mental; Promoção da saúde.

\begin{abstract}
This study aims to report the experience of teaching meditation online as a practice that promotes mental health during the social isolation of the COVID-19 pandemic. This is an experience report on one of the activities developed by the Extension Project for Mental Health Promotion Strategies at Universidade Federal Fluminense. Due to the COVID-19 pandemic, actions have been carried out remotely, in this case, meditation. Two cycles of Abundance meditation and two cycles of Ho'oponopono, with 21 days in each cycle, were performed. The abundance meditation activities were attended by 225 people, and the Ho'oponopono activities were attended by 357 people. Feedback from participants about the experience brought the perception of improvement in anxiety and fear of contamination by COVID-19, in addition to deep learning focused on self-knowledge. It was possible to conclude that meditation is a relevant resource for promoting mental health, especially in periods of social distance.
\end{abstract}

Keywords: Meditation; Quality of life; Mental health; Health promotion. 


\begin{abstract}
Resumen
Este estudio tiene como objetivo reportar la experiencia de enseñar meditación en línea como una práctica que promueve la salud mental durante el aislamiento social de la pandemia COVID-19. Este es un relato de experiencia sobre una de las actividades desarrolladas por el Proyecto de Extensión de Estrategias de Promoción de la Salud Mental en la Universidade Federal Fluminense. Debido a la pandemia de COVID-19, se han realizado acciones de forma remota, en este caso, meditación. Se realizaron dos ciclos de meditación de Abundancia y dos ciclos de Ho'oponopono, con 21 días en cada ciclo. A las actividades de meditación de la abundancia asistieron 225 personas ya las actividades de Ho'oponopono asistieron 357 personas. Los comentarios de los participantes sobre la experiencia trajeron la percepción de mejora en la ansiedad y el miedo a la contaminación por COVID-19, además de un aprendizaje profundo enfocado en el autoconocimiento. Se pudo concluir que la meditación es un recurso relevante para promover la salud mental, especialmente en períodos de distanciamiento social.
\end{abstract}

Palabras clave: Meditación; Calidad de vida; Salud mental; Promoción de la salud.

\title{
1. Introdução
}

A saúde mental é reconhecida pela Organização Mundial de Saúde (OMS), como um estado de completo bem-estar físico, mental e social, por isso, pensar na promoção da saúde mental como recurso para qualidade de vida, é preciso. A vida acadêmica desencadeia estresse, tanto para os alunos, quanto aos docentes e demais trabalhadores, devido às metas a serem alcançadas. Em períodos em que o distanciamento social é exigido, as práticas de promoção da saúde presenciais ficam impedidas, o que instiga profissionais a se readaptarem (Gouveia et al., 2020).

Os danos psicológicos aumentaram, devido ao medo do risco de contaminação por COVID-19, bem como pelo aumento do desemprego e a insegurança do futuro quanto a pandemia. A OMS aponta o aumento dos índices de suicídio, depressão, preocupação, medo, ansiedade, violência doméstica, fragilidade das redes de proteção e uso abusivo de álcool e outras drogas. É o que mostra um estudo realizado pela Universidade do Estado do Rio de Janeiro (UERJ) e publicado pela revista The Lancet. De acordo com o artigo, os casos de depressão aumentaram $90 \%$ e o número de pessoas que relataram sintomas como crise de ansiedade e estresse agudo mais que dobrou entre os meses de março e abril do ano de 2020 (Filgueira \& Stults-Kolehmainen, 2020).

Sentimentos de incertezas e instabilidades emocionais são amplificadas em razão da pandemia, situações que deixam a saúde mental mais comprometida, e assim, atividades que proporcionem um melhor gerenciamento da saúde mental e potencializem o bem-estar psicológico devem ser consideradas (Oliveira et al., 2020).

Neste sentido, têm-se as Práticas Integrativas e Complementares (PIC's) como modelo de cuidado milenar promotoras da saúde mental. As terapias integrativas valorizam o sujeito em suas subjetividades, emoções, pensamentos e peculiaridades, em todo o seu contexto. A Medicina Tradicional e Complementar (MTC), principalmente a chinesa, possui um conjunto de práticas terapêuticas que visualizam o indivíduo na sua integralidade: corpo físico, mente e espírito. Buscam promover a saúde, utilizando-se de meios naturais de tratamento (Teles Júnior, 2016).

A utilização das PIC's está embasada na Política Nacional de Práticas Integrativas e Complementares (PNPIC), como abordagens que buscam estimular os mecanismos naturais de prevenção de agravos e de recuperação da saúde por meio de tecnologias eficazes e seguras, com ênfase na escuta acolhedora, no desenvolvimento do vínculo terapêutico e na integração do ser humano com o meio ambiente e a sociedade (Brasil, 2015).

Em situações emocionais desenvolvidas em períodos de pandemia, essas terapias podem contribuir para o restabelecimento das emoções, aliviando o estresse e a ansiedade vivenciada por muitas pessoas. Dessa forma, surgem como possibilidade de enfrentamento ao isolamento social por meio da vivência de práticas corporais que poderão ser disponibilizadas para o acompanhamento on-line, com instruções apropriadas de algumas técnicas para a utilização em domicílios, sendo realizadas individualmente (Oliveira et al., 2020). Neste contexto, inclui-se a arteterapia, a meditação, a musicoterapia, o yoga, entre outros. 
Em virtude do exposto, desenvolveu-se um projeto de extensão na Escola de Enfermagem da Universidade Federal Fluminense (UFF), localizada em Niterói - Rio de Janeiro (RJ), com intuito de promover a saúde mental dos estudantes e docentes, possibilitando o autoconhecimento pela autorreflexão, com estratégias para modos mais saudáveis de vida. O projeto tem como título "Promoção da saúde mental dos estudantes e professores universitários", no qual são oferecidas atividades semanais, promotoras da saúde mental.

O referido projeto iniciou-se há oito anos, sendo oferecido primeiramente para os estudantes de enfermagem. Em seguida, atendendo a solicitação dos alunos de outros cursos, o projeto foi ampliado para todos, inclusive para residentes, mestrandos e doutorandos, tendo como justificativa a convivência no ambiente acadêmico, portanto, no mesmo ambiente estressor.

Com o surgimento da pandemia por COVID-19 e a necessidade do isolamento social, o projeto teve continuidade virtualmente e foi estendido também aos trabalhadores da UFF, seus familiares e amigos, tendo em vista que a realidade vivenciada impactou de maneira direta a vida de todos, afetando diretamente a saúde mental das pessoas.

Assim, o objetivo desse estudo é relatar a experiência do ensino da meditação on-line como prática promotora da saúde mental durante o isolamento social devido a pandemia por COVID-19.

\section{Metodologia}

Trata-se de um estudo descritivo do tipo relato de experiência, a partir das vivências da prática de meditação através de um projeto de extensão iniciado na Escola de Enfermagem da UFF. Refletiu-se acerca da qualidade de vida, bem-estar e saúde mental em tempos de pandemia por COVID-19, bem como sobre a possibilidade de adaptação das práticas para a continuidade das atividades promotoras de saúde mental. A etapa reflexiva foi fundamentada na formulação discursiva através das vivências práticas das autoras e dos conceitos teóricos sobre PIC's, e de maneira mais profunda, na meditação.

As experiências e ações relatadas aconteceram de forma virtual, entre os meses de maio e setembro de 2020 , tendo como sujeitos participantes os alunos, professores e servidores técnico-administrativos do projeto de extensão da Universidade Federal Fluminense (UFF), além de seus familiares e amigos. Neste sentido, esse relato é apresentado em dois momentos, a saber: "Readaptação do projeto de extensão durante a pandemia por COVID-19" e "Promovendo saúde mental em tempos de distanciamento social".

O estudo foi realizado de acordo com as recomendações estabelecidas pela Resolução n ${ }^{\circ}$ 510, de 07 de abril de 2016 (Art. $1^{\circ}$ ), que dispõe sobre as normas aplicáveis a pesquisas em Ciências Humanas e Sociais, e delibera que as pesquisas que objetivam o aprofundamento teórico de situações que emergem espontânea e contingencialmente na prática profissional, desde que não revelem dados que possam identificar o sujeito, não serão registradas nem avaliadas pelo sistema CEP/CONEP (Resolução 510/16, I, VII).

\section{Resultados e Discussão}

\subsection{Readaptação do projeto de extensão durante a pandemia por COVID-19}

De modo a dar continuidade ao projeto de extensão de forma virtual, a meditação foi a prática integrativa e complementar eleita, sendo oferecida de modo on-line, em ciclos com duração de 21 dias. A escolha se deu por estudos e prática pessoal das autoras, onde perceberam que a meditação minimiza os fatores causadores do estresse, podendo ser praticada em grupo ou individualmente, promovendo a saúde e o bem estar biopsicossocial e espiritual. Segundo Desai et al., (2021) programas de meditação on-line podem ser eficazes tanto pelo amplo público, quanto pela possibilidade de incorporar a prática ao estilo de vida e ao dia a dia. 
Assim, em maio de 2020 foi oferecido o primeiro ciclo de 21 dias de meditação on-line com boa aceitação dos participantes, aderência dos acadêmicos, docentes e demais pessoas inseridas no projeto. Ao término desse período, os participantes, de modo geral, referiram que foram impactados positivamente pela prática, e diante de tal fato, solicitaram outro ciclo de meditação, que começou em junho do mesmo ano.

Desta forma, a experiência aqui relatada foi realizada com dois tipos distintos de meditações, tendo como referenciais: Meditação da Abundância de Deepak Chopra e Meditação do Ho'oponopono de Carole Berger. O tempo de execução de cada atividade proposta compunha em média 30 minutos por dia. Ademais, para a execução de cada tipo de meditação foram necessários 84 dias totais de atividade, sendo, 42 dias para a Meditação de Deepak Chopra ( 2 ciclos de 21 dias) e 42 dias para Ho'oponopono (2 ciclos de 21 dias).

A meditação do Deepak Chopra explora o significado completo da consciência de prosperidade e abundância, afirmando que é o fluxo da generosidade do universo que conduz a riqueza material e espiritual para nossas vidas. $\mathrm{O}$ entendimento da consciência da prosperidade vai para além do senso comum, onde associa a prosperidade ao sucesso financeiro. Na meditação, aprende-se atitudes mentais necessárias para alcançar o conhecimento e o autoconhecimento (Chopra, 2018).

Por conseguinte, a meditação do Ho'oponopono tem como foco a prática do perdão e gratidão conforme antigos ensinamentos havaianos que compreenderam que somos pura energia materializada, e que se conectar com os ensinamentos do Ho'oponopono significa viver cada momento no mundo das energias. Nesta meditação trabalhou-se a percepção da gratidão, do fluxo do perdão na vida, bloqueios, medos, crenças limitantes, sombras, reflexões familiares e da infância. A compreensão da lei da gratidão e sua incorporação em nossa vida proporcionam as chaves para a resolução de muitos conflitos internos (Berger, 2020).

Acredita-se que o ser humano tende a se colocar em diversas situações, todos os dias, como um ciclo enlouquecedor de afazeres sem significados ou vontades. Neste sentido, o entendimento dos processos que levam ao padecimento ganha novos significados, uma vez que esses ciclos de frustrações levam ao acúmulo de estresse, fadiga, raiva e depressão. A meditação, em contrapartida, convoca para a harmonia e responsabilidade individual de cada um sobre o processo de saúde-doença, trazendo a consciência da identidade (Chopra, 2018).

Os grupos foram criados a convite da coordenadora do projeto pelas redes sociais e o link de acesso foi compartilhado no intuito de agregar maior quantidade de participantes. Evidencia-se que antes de iniciar as atividades algumas regras foram estabelecidas, como: separar um caderno próprio para realização das tarefas e utilizar o grupo on-line apenas para informar sobre a conclusão da meditação e das atividades propostas. Diariamente, após a postagem das meditações e atividades do grupo, os participantes teriam 24 horas para concluir as referidas atividades e a seguir, deveriam postar apenas a informação: Dia 01 feito; Dia 02 - feito, e assim sucessivamente.

As atividades do ciclo de abundância foram compartilhadas como orientadas pelo programa de Deepak Chopra, com a participação de 225 pessoas. Os ciclos do Ho'oponopono contaram com a participação de 357 pessoas. Ressalta-se que muitos participantes quiseram repetir o mesmo ciclo com a justificativa de sentir necessidade de aprofundar o conhecimento e aperfeiçoar a prática meditativa, o que se considera relevante, tendo em vista que diversos indivíduos nunca haviam praticado a meditação e mesmo aqueles que já praticavam, referiram sentir-se bem com as práticas naquele contexto.

Ao final dos referidos ciclos, foi solicitado que os participantes enviassem o feedback sobre a experiência, em formato de vídeo, áudio ou escrito, sendo evidenciado a viabilidade de meditar, a percepção quanto a melhora da ansiedade e o medo da contaminação por COVID-19, além dos relatos de aprendizado profundo com foco significativo no autoconhecimento. 


\subsection{Promovendo saúde mental em tempos de distanciamento social}

Durante os 21 dias de cada ciclo, foram enviados vídeos com as práticas de meditação, o mantra do dia, assim como as atividades que eram disparadoras para momentos reflexivos e promotores da saúde mental. Como feedback foi possível perceber que terapias integrativas, dentre elas a meditação, surgem como possibilidade terapêutica que beneficiam a saúde no combate aos agentes estressores decorrentes de períodos de isolamento social. Pesquisas corroboram com esses resultados positivos na redução dos níveis de ansiedade, de sintomas depressivos, de estresse, de alívio da dor e da compulsão alimentar (Kim et al., 2013; Kemper et al., 2011).

As terapias integrativas no período da quarentena, imposta pela pandemia, favorecem o reequilíbrio das emoções, a diminuição da ansiedade e o controle do medo. Parte considerável das PIC's pode ocorrer de maneira remota, de acordo com o isolamento exigido no momento, devendo ser utilizada diante do impacto emocional e físico decorrente da pandemia. No entanto, sabe-se que tais práticas não devem substituir o tratamento convencional, porém, a sua utilização complementa o cuidado, a partir de orientações de pessoas capacitadas para cada situação (Brasil, 2020).

A prática frequente de meditação, tanto semanal como para aquelas pessoas com experiência de anos, é preditora de bem-estar subjetivo, ou seja, é possível de ser observado um melhor índice de afetos positivos e satisfação com a vida tanto nas pessoas que tem a meditação como recurso promotor de saúde mais vezes por semana em um espaço de tempo recente, como naquelas pessoas que o fazem há mais tempo como rotina. Evidencia-se também, que para ambos observa-se menores níveis de afetos negativos, o que pode ser um fator incentivador para o investimento na prática (Hernández \& Martins, 2020).

Foi realizada uma pesquisa com estudantes dos cursos de Medicina, Enfermagem e Fonoaudiologia de uma universidade pública de São Paulo, a partir de um curso eletivo focado na meditação de atenção plena e compaixão, em que os estudantes relataram uma redução na ansiedade e a diminuição de escores negativos, principalmente relacionados ao medo e a hostilidade, com a imersão nas práticas meditativas. Os indivíduos afirmaram que passaram a conseguir viver mais o momento presente com menor tentativa de pensar ou antecipar os acontecimentos do futuro, entendendo a importância de lidar com as emoções ou invés de reprimi-las (Araujo et al., 2020).

Esse estudo corrobora com Menezes et al. (2012), os quais realizaram uma pesquisa que objetivou investigar o interesse dos alunos de uma universidade federal em participar de um treino extracurricular de seis semanas de meditação, bem como suas motivações para a participação. Neste sentido, as principais motivações foram direcionados para os benefícios emocionais, como a redução de estresse/ansiedade, qualidade de vida/bem-estar mental, autoconhecimento/autocontrole, além do gosto e interesse pessoal pelo assunto, seja por um interesse já existente ou para conhecer por já ter ouvido ou lido a respeito dos benefícios. Com isso, entende-se que essa prática pode ser uma alternativa de assistência estudantil no contexto universitário, sendo destinada tanto para grupos clínicos como não clínicos.

Assim, é importante está atento para a importância de tais práticas nesse ambiente universitário, como também nos ambientes de trabalho. A prática meditativa pode fornecer subsídios importantes ancorados em suporte emocional, melhora da atenção e concentração, tendo em vista as queixas desse público de refletem na dificuldade em viver o presente sempre buscando o controle não somente do hoje, mas do futuro e o cultivo dos sentimentos hostis. Praticar a meditação se apresenta como auxílio no manejo das emoções, principalmente na modulação dos sentimentos desconfortáveis (Araujo et al., 2020).

Cuidar do corpo e da mente se torna complexo, pois não envolve somente o orgânico, mas também o emocional, mental e espiritual. Períodos de pandemia fazem emergir sentimentos de angústias e incertezas que passam a interferir na saúde, gerando doenças, conflitos e medos, ocasionando um colapso mental, levando ao corpo à sensação de exaustão. As PIC's surgem como possibilidade de enfrentamento ao isolamento social por meio da vivência de práticas corporais que poderão ser disponibilizadas para o acompanhamento por meio on-line, com instruções apropriadas para a utilização em domić́lio de algumas técnicas realizadas individualmente (Oliveira et al., 2020). 
Estudos mostram que algumas PIC's afetam positivamente a qualidade de vida, o sono, a dor, o estado emocional, o bem-estar e o apoio social de seus praticantes (McFeeters et al., 2016; Ferreira et al., 2017). Os indivíduos participantes confirmaram que a meditação estimulou o autocuidado, o cuidado com o outro, a corresponsabilização no processo saúde-doença e a ética humana na integração com a sociedade e a natureza, em uma perspectiva criativa e participativa.

Por fim, torna-se importante frisar a participação dos docentes de magistério superior nas atividades propostas. Dessa maneira, 30 professores participaram ativamente, e alguns participaram mais de uma vez em cada ciclo, revelando uma predisposição para práticas promotoras de saúde mental no trabalho.

Os docentes tem sido afetados grandemente pelo adoecimento mental, e patologias relacionadas aos transtornos mentais e comportamentais têm sido contempladas como as maiores queixas de saúde e também de índices de afastamento no ambiente de trabalho, gerando sofrimento e sendo um importante problema de saúde pública (Campos et al., 2020).

Neste sentido, agir junto aos profissionais na busca por estratégias de promoção da saúde mental nesse espaço, possibilitará promover e prevenir o adoecimento mental, principalmente ao entender que a universidade é um lugar com sentido de comunidade, onde estão docentes, técnico-administrativos em educação, terceirizados, discentes e todos àqueles que direta ou indiretamente podem ser atravessados em um cuidado contínuo no trabalho.

A experiência da meditação pode agregar benefícios aos cuidados de saúde, principalmente no que diz respeito à promoção da saúde mental, uma vez que é um método que permite explorar o cultivo das qualidades e capacidades que resultam em um bem-estar físico e psicológico, promovendo o autoconhecimento e o manejo dos fatores estressores diários, em que este manejo, por sua vez, surge como uma consequência da prática meditativa (Votto \& Carvalho, 2019).

Por fim, é importante mencionar que a relação da escola, universidade e trabalho com práticas como meditação, que desenvolvem os aspectos espirituais tende a despertar sentimentos de empatia, responsabilidade, sensibilidade, compaixão, esperança, não possuindo relação com as religiões, mas sim alcançando um processo de contemplação e respeito à vida. Neste sentido, a meditação propicia ao sujeito a reflexão quanto as próprias ações, e que, a partir do desenvolvimento do autocuidado, o olhar para si e para o outro torna-se mais aguçado, empoderando indivíduos e promovendo a saúde mental individual e entre os pares (Cossia \& Andrade, 2020).

\section{Considerações Finais}

Foi possível verificar que os participantes conseguiram se conectar com a prática da meditação e relataram a diminuição do estresse, da ansiedade e das aflições que todos são expostos no cotidiano, principalmente no momento atual de pandemia por COVID-19. Através dessa reflexão, entende-se que os efeitos positivos apresentados pela utilização da meditação como uma das Práticas Integrativas e Complementares no período de isolamento e/ou distanciamento social ocasionado pela pandemia pode ser considerada como um complemento ao cuidado e autocuidado.

A execução da experiência da meditação ajudou na compreensão dos participantes a respeito da importância de meditar e da consequente promoção da saúde mental, contemplando todos os seus benefícios e suas dimensões: física, mental, social e -espiritual. Considera-se que as terapias integrativas e complementares produzem aumento da autoestima, redução dos agentes estressores, sensação de bem-estar, diminuição de sentimentos negativos, que são por vezes, apresentados em decorrência da necessidade de se manter em isolamento.

A realização das atividades disparadoras foi desafiadora, mas importante e necessária para a maioria dos participantes, evidenciando-se nas gravações de voz e vídeos recebidos ao termino dos ciclos, onde foi possível constatar a tranquilidade e o conforto proporcionados pela prática da meditação, bem como demonstrou o fortalecimento da gratidão familiar e dos desbloqueios de crenças. Conclui-se, portanto, que a meditação é um excelente recurso para promoção da saúde mental, principalmente na época atual, contribuindo para o gerenciamento das emoções como uma grande ferramenta de cuidado. 


\section{Agradecimentos}

A todos os indivíduos participantes do projeto de extensão, que compartilharam saberes e aprendizados, e acreditam que as atividades promotoras de saúde mental podem atuar de modo transformador.

\section{Referências}

Araujo, A. C., Santana, C. L., Kozasa, E. H., Lacerda, S. S., \&Tanaka, L. H. (2020). Efeitos de um curso de meditação de atenção plena em estudantes da saúde no Brasil. Acta Paul Enferm, 33, 1-9. doi: 10.37689/acta-ape/2020AO0170.

Berger, C. (2020). Ho ’oponopono: a antiga prática havaiana da gratidão e do perdão. Pensamento.

Brasil. (2015). Política nacional de práticas integrativas e complementares no SUS: atitude de ampliação de acesso. (2a. ed.). Ministério da Saúde.

Brasil. (2020). Recomendação No 041 (2020, maio 21). Recomenda ações sobre o uso das práticas integrativas e complementares durante a pandemia da Covid19. http://conselho.saude.gov.br/recomendacoescns/1192-recomendacao-n-041-de-21-de-maio-de- 2020.

Campos, T. C., Véras, R. M., \& Araújo, T. M. (2020) Trabalho docente em universidades públicas brasileiras e adoecimento mental: uma revisão bibliográfica. Revista Docência do Ensino Superior, 10: e015193. 10.35699/2237-5864.2020.15193.

Chopra, D. (2018). Criando prosperidade: 26 passos para uma vida mais rica e abundante. Alaúde.

Cossia, T., \& Andrade, M. F. R (2020). Contribuições da meditação em âmbito escolar. Interfaces da Educ., 11(31), 153 - 176. 10.26514/inter.v11i31.4111.

Desai, K., Gupta, P., Parikh, P \& Desai, A. (2021). Impacto of virtual heartfulness meditation programo on stress, quality of sleep, and psychological wellbeing during the COVID-19 pandemic: a mixed-method study. Int J Environ ResPublic Health, 18, 11114. doi.org/10.3390/ijerph18211114.

Ferreira, J. A., Monteiro, A. D. C., Lima, N. B. A., \& Souza, P. T. L. (2017). Práticas não convencionais em saúde por familiares e vínculos afetivos de pacientes críticos. Revista de Pesquisa: Cuidado é Fundamental Online, 9(1), 200-207. http://www.seer.unirio.br/index.php/cuidadofundamental/article/view/5363.

Filgueira, A., \& Stults-Kolehmainen, M. (2020). Fatores associados a mudanças nos resultados de saúde mental entre brasileiros em quarentena devido ao COVID-19. medRxiv preprint.: https://www.medrxiv.org/content/10.1101/2020.05.12.20099374v2.

Gouveia, A. O., Silva, H. R. S., \& Batista Neto, J. B. S. (2020). Saúde mental em tempos de COVID-19: construção de cartilha educativa com orientações para o período de pandemia. Enfermagem em Foco, 11(1), 168-173. http://revista.cofen.gov.br/index.php/enfermagem/article/view/3600.

Hernandéz, D. N., \& Martins, G. H. (2020). Bem-estar subjetivo em praticantes e não praticantes de meditação. Interação em Psicologia, 24(01), 31-41. http://dx.doi.org/10.5380/psi.v24i1.64925.

Kemper, K., Bulla, S., Krueger, D., Ott, M. J., McCool, J. A., \& Gardiner, P. (2011). Nurses experiences, expectations, and preferences for mind-body practices to reduce stress. BMC Complementary Alternative Medicine, 11, 11-26. https://pubmed.ncbi.nlm.nih.gov/21481259/.

Kim, J. H., Yang, H., \& Schroeppl, S. (2013). Peilot studies examining the effects of kouk sun do on university students with anxiety symptoms. Stress Health, 29(2), 99-107. https://www.researchgate.net/publication/225275812.

Menezes, C. B., Fiorentin, B \& Bizarro, L. (2012). Meditação na universidade: a motivação de alunos da UFRGS para aprender meditação. Rev Sem Assoc Bras de Psic Esc e Educ, 16(2), 307-315, Retrieved 13 Jan, 2022, from: https://www.scielo.br/j/pee/a/vyRqD9wJVKHfmQmxJrvx7bF/?format=pdf\&lang=pt.

McFeeters, S., Leeanne Pront, L., Cuthbertson, L., \& King, L. (2016). Massage, a complementary therapy effectively promoting the health and well-being of older people in residential care settings: a review of the literature. International Journal of Older People Nursing, 11(4), 266-283. 10.1111/opn.12115.

Oliveira, E. N., Costa, M. S. A., Marques, N. S., Lomeo, R. C., Nascimento, P. I. F. V., Rodrigues, C. S., Andrade, C. S. G., \& Moreira, R. M. M. (2020). Projeto vida em quarentena: estratégia para promoção da saúde mental de enfermeiros diante da COVID-19. Enfermagem em Foco, 11 (1) http://revista.cofen.gov.br/index.php/enfermagem/article/view/3741/820.

Oliveira, F. P., Lima, M. R. S., \& Farias, F. L. R. (2020). Terapias integrativas e complementares em situações emocionais na pandemia do COVID-19. Rev Interdisciplinar, https://revistainterdisciplinar.uninovafapi.edu.br/index.php/revinter/article/view/1778/pdf_462.

Teles Júnior, E. (2016). Práticas integrativas e complementares em saúde, uma nova eficácia para o SUS. Estudos avançados, 30(86), 99-112. 10.1590/S010340142016.00100007 .

Votto, G. G., \& Carvalho, H. W. (2019). Bem-estar psicológico e meditação: um estudo associativo. Estudos Interdisciplinares em Psicologia, 10(3), 60-75. 10.5433/2236-6407.2019v10n3p60. 